\title{
Dynamic Characterisation of Base-Isolated Structures Using Analytical Shear-Beam Model
}

\author{
Vasant A. Matsagar \\ Department of Civil Engineering, Lawrence Technological University, Southfield, 48075 Michigan, USA \\ R. S. Jangid \\ Department of Civil Engineering, Indian Institute of Technology Bombay, Powai, 400076 Mumbai, India
}

(Received 18 May 2006; accepted 18 July 2006)

\begin{abstract}
An analytical investigation of the dynamic characteristics of a shear beam structure supported on linear laminated rubber bearings is carried out. The superstructure is modelled as a continuum shear beam. The base-raft forming part of the base-isolated building is modelled as mass lumped at the lower end of the shear beam, while the effective linear stiffness is assumed for the bearings. In addition to the base-isolated shear beam, closed-form solutions for two auxiliary shear beams with free-spring and unconstrained end conditions are obtained for comparison. The frequencies of the base-isolated shear beam are found to fall into a range bounded by those of the two auxiliary shear beams, forming two limiting cases of base-isolated shear beams. It is seen that the higher modes of the base-isolated beam are practically identical with those of the unconstrained shear beam, and therefore, orthogonal to the horizontal ground motion. The contribution of higher modes towards the response of superstructure is therefore negligible.
\end{abstract}

\section{INTRODUCTION}

Base isolation, the use of laminated rubber bearings, or similar devices, at the base of structures to isolate it from ground is a concept that is commonly adopted concept for the protection of structures from devastating earthquakes. ${ }^{1-3}$ It has been shown in previous analyses that the seismic response of base-isolated structures can be obtained by modelling the superstructure as rigid blocks supported on seismic isolation systems. ${ }^{4-12}$ The rigid block assumption results in a simplified single-degree-of-freedom system which is suitable for the preliminary design of base-isolated structures. However, the results that are obtained based on the rigid block assumption are limited to stiff structures where the influence of flexibility in the superstructure is negligible. Using discrete models, the effects of superstructure flexibility on the response of baseisolated structure and its attached equipments were investigated previously. ${ }^{8,13}$ A numerical investigation was carried out in an another comparative study of performances of various base isolators for shear beam type structures. ${ }^{14} \mathrm{~A}$ study is consequently indispensable to illustrate the dynamics involved, to characterise the base-isolated structures, and to demonstrate their behaviour during earthquake excitations analytically.

This paper focuses on the analytical investigations of the dynamic characteristics of the base-isolated continuum shear beam model subjected to a horizontal ground motion, and brings about some interesting observations. In addition to the base-isolated shear beam, two auxiliary shear beam models with free-spring and unconstrained end conditions are also examined. For comparison, the dynamic characteristics of the three types of shear beams are shown in closed-form expressions. The shifting of natural frequencies and the contribution of higher modes towards the response of superstructure is studied.

\section{ANALYTICAL SHEAR BEAM MODEL OF ISOLATED STRUCTURE}

One of the simplest, but important continuum models for building structures is the shear beam model. A building on base isolation systems is idealised here as a continuum in the form of a uniform shear beam. It is assumed that the baseisolated structure behaves elastically; the beams are rigid (due to in-plane diaphragm action of slabs), and columns do not deform axially. These assumptions are generally valid for typical base-isolated structures, because due to the insertion of isolators underneath these structures, the superstructure is isolated from the ground. The mass of the base-raft on top of the elastomeric rubber isolation systems is lumped at the lower end of the uniform shear beam while an elastic spring representing the equivalent effective stiffness of the isolation bearings is attached to the lumped base mass. The effects of the use of such linear equivalent stiffness of the isolators have been studied previously. ${ }^{15}$

The idealised system for the base-isolated shear beam model is shown in Fig. 1(a) when no external excitation is applied. Due to the earthquake ground motion, the baseisolated shear beam is deflected as shown in Fig. 1(b). Figures 1(c) and (d) show the configurations of two auxiliary shear beams, which are referred to as the unconstrained shear beam and the free-spring shear beam models, respectively.

The elastic stiffness of a shear beam is represented by $G A_{s}$, where $G$ is the modulus of rigidity and $A_{s}$ is the effective shear area, while the mass per linear length of the shear beam is assumed to be $\rho A$, where $\rho$ is the mass density and $A$ is the cross-sectional area. $A_{s}$ is related to $A$ such that $A_{S}=\kappa A$, where $\kappa$ is the shape factor. The shape factor $\kappa$ is an area reduction factor necessary to determine the effective shear area because the shear is not constant over the cross-section. The 